J. Math. Soc. Japan

Vol. 26, No. 1, 1974

\title{
Almost polar sets and an ergodic theorem
}

\author{
By Masatoshi Fukushima
}

(Received March 25, 1972)

\section{$\S 1$. Introduction.}

We will develop a potential theory for two Markov processes which are in duality and apply it to an extension of the Chacon-Ornstein ergodic theorem.

Let $X$ be a locally compact separable Hausdorff space and $m$ be a positive Radon measure on $X$. Consider standard Markov processes $\boldsymbol{M}=\left(X_{t}, P_{x}\right)$ and $\hat{\boldsymbol{M}}=\left(\hat{X}_{t}, \hat{P}_{x}\right)$ which are in duality with respect to $m$ in the sense that the equality

$$
\left(f, T_{t} g\right)=\left(\hat{T}_{t} f, g\right), \quad t>0,
$$

holds for any non-negative Borel functions $f$ and $g$ on $X$. Here $T_{t}$ (resp. $\hat{T}_{t}$ ) is the semi-group associated with $\boldsymbol{M}$ (resp. $\hat{\boldsymbol{M}}$ ) and $(f, g)$ is the integral $\int_{\boldsymbol{X}} f(x) g(x) m(d x)$. The quantities relative to the dual process $\hat{\boldsymbol{M}}$ are denoted with a and designated by the prefix co-. Notice that the present duality is much weaker than that of Blumenthal-Getoor [2; VI] and we do not assume the absolute continuity of resolvents or transition probabilities.

A set $A \subset X$ is said to be almost polar if there is a Borel set $B$ such that $A \subset B$ and

$$
P_{x}\left(\sigma_{B}<+\infty\right)=0 \quad \text { for } m \text {-a. e. } x \in X,
$$

where $\sigma_{B}$ is the hitting time inf $\left\{t>0 ; X_{t} \in B\right\}$. "Quasi-everywhere" or "q. e." will mean "except on an almost polar set".

Recently the notion of almost polarity was employed independently by S. Port and C. Stone [12] for additive processes with $m$ being the Haar measure and by the author [8] for general $m$-symmetric Markov processes whose associated Dirichlet spaces are regular ${ }^{1)}$. In both cases, almost polar sets were identified with the sets of $\lambda$-capacity zero, the $\lambda$-capacity being defined suitably according to the respective situations. When $\boldsymbol{M}$ is the Brownian motion on $R^{3}$, the almost polar set is just the set of the Newtonian outer capacity zero.

1) Almost polar sets are called "essentially polar" in [12] and "polar" in [8]. 
In $\S 2$, we will study almost polar sets together with q.e. finely continuous functions and present some fundamental properties that they possess. Assertions $(\mathbf{i}) \sim(\mathbf{i x})$ of $\S 2$ are the generalizations of those established in $[8 ; \S 3,4]$, while $(\mathbf{x}) \sim(\mathbf{x i v})$ are our versions of those in Blumenthal-Getoor [2; VI]. The second assertion states that each almost polar set is $m$-negligible. But the converse is not necessarily true. Proposition (viii) asserts that the resolvent of $\boldsymbol{M}$ is absolutely continuous with respect to $m$ if and only if each almost polar set is semipolar. The final assertion states that the next two conditions $\left(\boldsymbol{C}_{1}\right)$ and $\left(\boldsymbol{C}_{1}^{\prime}\right)$ are equivalent.

$\left(\boldsymbol{C}_{1}\right)$ Each semipolar set is almost polar,

$\left(\boldsymbol{C}_{1}^{\prime}\right)$ A function is q. e. finely continuous if and only if it is q. e. cofinely continuous.

In particular, condition $\left(\boldsymbol{C}_{1}\right)$ is met when $\boldsymbol{M}$ is $m$-symmetric $(\boldsymbol{M}=\hat{\boldsymbol{M}})$.

In $\S 3$, we will prove under the assumption $\left(\boldsymbol{C}_{1}\right)$ that, for any bounded Borel $f, g \in L^{1}(X ; m), g \geqq 0$, the ratio

$$
\frac{\int_{0}^{t} T_{s} f(x) d s}{\int_{0}^{t} T_{s} g(x) d s}
$$

converges, as $t \rightarrow+\infty$, to a finite limit q. e. on the set where the denominator becomes eventually positive. The novelty of this generalization of the Chacon-Ornstein theorem is that the original statement of a.e. convergence is strengthened to q. e. convergence. Since almost polarity and $m$-negligibility are equivalent, however, in the cases of discrete time Markov processes, the two notions have not been distinguished in ergodic theory so far. Our key step is to generalize Brunel's ergodic inequality following the line of A. Garsia [10]. Brunel's inequality combined with a potential theoretic result of $\S 2$ will immediately implies q. e. convergence of the ratio (1.3).

We note that, in other fields of analysis, especially in Fourier analysis and the boundary limit theorem, we often encounter this kind of phenomena of the transfer from a. e. to q. e. (cf. Carleson's monograph [4]).

Our ratio limit theorem can be applied to an ergodic decomposition of the state space. In $\S 4$ we will show that the conservative (resp. dissipative) part of our decomposition consists, after a suitable modification by a polar set, just of finely recurrent (resp. transient) points in the sense of Azema, Kaplan-Duflo and Revuz [1], assuring further that the conservative part is not only $T_{t}$-invariant but also sample path-invariant. An additional absolute continuity condition will be imposed in $\S 4$, for we will have to use a theorem of J. L. Doob [6] concerning the quasi Lindelöf property of the fine topology. 


\section{§2. Potential theory for $M$ and $\hat{M}$.}

Our assertions will be listed up.

(i) Let $E$ be universally measurable. The next three conditions are equivalent.

( $\alpha) \quad m(E)=0$.

( $\beta) ~ p(t, x, E)=0$ m-a.e. $x \in X$, for each $t>0$.

(r) $G_{\alpha}(x, E)=0 m$-a.e. $x \in X$, for every $\alpha>0$ (equivalently for some $\alpha>0$ ). Here $p(t, x, E)$ (resp. $\left.G_{\alpha}(x, E)\right)$ is the transition probability (resp. the resolvent kernel) of the process $\boldsymbol{M}$.

Proof. We only show the implication $(\beta) \Rightarrow(\alpha)$ :

$$
0=\lim _{t \downarrow 0} \int_{X} T_{t} I_{E}(x) m(d x)=\lim _{t \downarrow 0} \int_{X} \hat{T}_{t} 1(x) I_{E}(x) m(d x) \geqq \int_{X} I_{E}(x) m(d x)
$$

where $I_{E}$ denotes the indicator of $E$.

(ii) If $N$ is almost polar, then $m(N)=0$.

Proof. There is a Borel almost polar set $E \supset N$. Then $E$ satisfies ( $\beta$ ). Hence $m(E)=0$.

(iii) Let $A$ be a Borel set and put

Then we have

$$
\begin{aligned}
& T_{t}^{0} f(x)=E_{x}\left(f\left(X_{t}\right) ; t<\sigma_{A}\right), \\
& H_{A}^{\alpha} f(x)=E_{x}\left(e^{-\alpha \sigma_{A}} f\left(X \sigma_{A}\right)\right) .
\end{aligned}
$$

$$
\begin{aligned}
& \left(f, T_{t}^{0} g\right)=\left(\hat{T}_{t}^{0} f, g\right), \quad t>0, \\
& \left(f, H_{A}^{\alpha} G_{\alpha} g\right)=\left(\hat{H}_{A}^{\alpha} \hat{G}_{\alpha} f, g\right), \quad \alpha>0,
\end{aligned}
$$

for any non-negative Borel functions $f$ and $g$. Here $G_{\alpha}$ is the resolvent of $T_{t}$.

Proof. (2.2) is equivalent to (2.1). (2.1) was proved by Dynkin [7; Lemma 14.1] for the Brownian motion by making use of a method of time reversion. The same method has been extended to $m$-symmetric Markov processes in [8; Theorem 3.5]. The argument there is independent of the symmetry of $T_{t}$ and only the relation of duality (1.1) is enough to get (2.1) for open $A^{2}$. Next (2.1) for any Borel $A$ can be obtained just as in [2; pp. 262] by noticing that any semi-polar set (resp. cosemi-polar set) is of potential zero (resp. copotential zero) and hence $m$-negligible according to (i).

For a nearly Borel set $E \subset X$, we will write

$$
\left\{\begin{array}{l}
e_{E}^{\alpha}(x)=H_{E}^{\alpha} 1(x) \\
e_{E}(x)=H_{E}^{0+} 1(x)=P_{x}\left(\sigma_{E}<+\infty\right) .
\end{array}\right.
$$

2) Recently T. Watanabe [14] gave a quite different proof of the relation (2.2) for open sets by making use of a method of the balayage of excessive measures. 
Here are two consequences of the relation (2.2).

(iv) Almost polarity and almost copolarity are equivalent.

Proof. Let $E$ be Borel and almost polar. Then, for any $g \in C_{0}(X)$ (the space of continuous functions with compact supports), $0=\left(1, H_{E}^{\alpha} G_{\alpha} g\right)=$ $\left(\hat{H}_{E}^{\alpha} \hat{G}_{\alpha} 1, g\right)$. Hence $0=\hat{H}_{E}^{\alpha} \hat{G}_{\alpha} 1 \geqq \hat{H}_{E}^{\alpha} \hat{G}_{\beta} 1 m$-a. e. for all $\beta \geqq \alpha$. But then $\hat{e}_{E}^{\alpha}(x)=$ $\lim _{\beta \rightarrow+\infty} \beta \hat{H}_{E}^{\alpha} \hat{G}_{\beta} 1(x)=0$ for $m$-a. e. $x \in X$, proving that $E$ is almost copolar.

( $v)$ Let $A$ be nearly Borel and finely open. Suppose that a nearly Borel subset $E \subset A$ has the property that

$$
\hat{e}_{E}(x)=0 \quad m-a . e \text {. on } A .
$$

Then $E$ is almost polar.

PROof. If $E$ is nearly Borel, then there are, for a strictly positive function $h \in L^{1}(X, m)$, some Borel sets $E^{\prime}$ and $E^{\prime \prime}$ such that $E^{\prime} \subset E \subset E^{\prime \prime}$ and $P_{h \cdot m}\left(X_{t} \in E^{\prime \prime}-E^{\prime}\right.$ for some $\left.t \geqq 0\right)=0$, which means that $E^{\prime \prime}-E^{\prime}$ is almost polar. Hence it suffices to show the proposition (v) for a Borel set $E$.

Take any compactum $K \subset E$. Since $\hat{e}_{K}=0 \mathrm{~m}$-a.e. on $A$, we have $0=$ $\left(\hat{H}_{K}^{\alpha} \hat{G}_{\alpha} f, I_{A}\right)=\left(f, H_{K}^{\alpha} G_{\alpha} I_{A}\right)$ for any $f \in C_{0}(X)$. Therefore $H_{K}^{\alpha} G_{\beta} I_{A}=0 \mathrm{~m}$-a. e. for all $\beta>\alpha . \quad H_{K}^{\alpha}$ is supported by $K$ but $\lim _{\beta \rightarrow \infty} \beta G_{\beta} I_{A}(y)=1$ for $y \in K(\subset A)$ because $A$ is finely open. We get $e_{K}^{\alpha}(x)=0 m$-a. e. Now find, for a strictly positive $h \in L^{1}(X ; m)$, an increasing sequence of compact sets $K_{n} \subset E$ such that $\left(h, e_{E}^{\alpha}\right)$ $=\lim _{n \rightarrow \infty}\left(h, e_{K_{n}}^{\alpha}\right)$ to complete the proof.

Definition. A function $f$ defined q. e. on $X$ is called q.e. finely continuous if the following conditions are satisfied: there exists a nearly Borel almost polar set $B$ such that $X-B$ is finely open and $f$ is nearly Borel measurable and finely continuous on $X-B$.

(vi) If $f$ is q.e. finely continuous and if $f \geqq 0 m-a$.e. on $X$, then $f \geqq 0$ q.e. on $X$.

Proof. Let $B$ be the set appeared in the above definition of q. e. fine continuity of $f$. Then the set $A=(X-B) \cap\{x ; f(x)<0\}$ is nearly Borel and finely open. Since $m(A)=0, \hat{e}_{A}=0 m$-a. e. on $A$ trivially and $A$ is almost polar by $(\mathbf{v})$.

The following characterization of almost polar sets already appeared in [8; Theorem 3.12]. We say that a set $E$ is $\boldsymbol{M}$-invariant if $P_{x}\left(X_{t} \in E\right.$ for every $t \in[0, \zeta))=1$ for every $x \in E^{3}$.

(vii) $A$ set $N$ is almost polar if and only if there exists a Borel set $B \supset N$ such that $m(B)=0$ and $X-B$ is M-invariant.

Proof. Let $N$ be almost polar then there is a Borel set $B_{0} \supset N$ such that $e_{B_{0}}(x)=0 m$-a. e. Since $e_{B_{0}}$ is excessive, it is nearly Borel and finely continuous.

3) $\zeta$ is the life time of the process $\boldsymbol{M}$. 
Hence, by the previous assertion, $e_{B_{0}}(x)=0$ q. e., that is, except on some Borel almost polar set $B_{1}$. Apply the same argument to the function $e_{B_{0} \cup B_{1}}$. In this way, we get a sequence $B_{0}, B_{1}, \cdots, B_{k}, \cdots$ of Borel almost polar sets. It suffices to put $B=\bigcup_{k=0}^{\infty} B_{k}$.

Now we will give some criteria for the absolute continuity of the resolvent in terms of the relationship among almost polarity, polarity and semipolarity.

(viii) The following four conditions are mutually equivalent.

( $\alpha$ ) A set is almost polar if and only if it is polar.

( $\beta$ ) Any almost polar set is semipolar.

( $\gamma) \quad m$ is a reference measure for $\boldsymbol{M}$ : a set is of potential zero if and only if it is m-negligible.

(ס) $G_{\alpha}(x, \cdot)$ is absolutely continuous with respect to $m$ for each $\alpha>0$ and $x \in X$.

PRoOF. $(\gamma)$ and $(\delta)$ are equivalent in view of the first assertion (i). $(\alpha)$ implies $(\beta)$. Suppose that the condition $(\beta)$ is satisfied. Let $E$ be an $m$ negligible Borel set. Then $G_{\alpha}(x, E)=0 m$-a. e. $x \in X$, by virtue of (i). But $G_{\alpha}(\cdot, E)$ is $\alpha$-excessive and finely continuous. Hence $G_{\alpha}(x, E)=0$ q. e. by (vi) and moreover except on a semipolar set by the present assumption. Since any semipolar set is of potential zero, we have $G_{\alpha}(x, E)=\lim _{\beta \rightarrow \infty} \beta G_{\beta+\alpha} G_{\alpha} I_{E}(x)=0$, $x \in X$, arriving at $(\delta)$. Evidently $(\delta)$ implies $(\alpha)$. The proof is finished.

REMARK $1^{\circ}$. Assertion (viii) is a generalization of [8; Theorem 3.13]. Combining (vii) and (viii), we get the following criterion: $G_{\alpha}(x, \cdot)$ is not absolutely continuous with respect to $m$ for some $\alpha>0$ and $x \in X$ if and only if there exists an $m$-negligible Borel set $E$ such that $X-E$ is $\boldsymbol{M}$-invariant but $E$ is not thin.

$2^{\circ}$. In the case that $\boldsymbol{M}=\hat{\boldsymbol{M}}$, those conditions in (viii) are also equivalent to the following one $(\varepsilon)[9]$.

( $\varepsilon$ ) The transition probability $p(t, x, \cdot)$ is absolutely continuous with respect to $m$ for each $t>0$ and $x \in X$.

The next proposition says that we can reduce the nearly Borel measurability of q. e. finely continuous functions to the Borel measurability.

(ix) A function $f$ is q.e. finely continuous if and only if there exists a Borel almost polar set $B$ such that $X-B$ is $\boldsymbol{M}$-invariant and $f$ is Borel measurable and finely continuous on $X-B$.

Proof. Let $f$ be q. e. finely continuous. Then by the definition and (vii), there is a Borel almost polar set $B_{0}$ such that $X-B_{0}$ is $\boldsymbol{M}$-invariant and $f$ is nearly Borel and finely continuous on $X-B_{0}$. For a fixed natural number $M$ we define the trancated function $f^{M}$ of $f$ by $f^{M}=(f \wedge M) \vee(-M)$ on $X-B_{0}$. We extend $f^{M}$ by setting its value to be zero on $B_{0}$. By the fine continuity of $f^{M}$ on $X-B_{0}$, we have 


$$
\lim _{n \rightarrow \infty} n G_{n} f^{M}(x)=f^{M}(x), \quad x \in X-B_{0} .
$$

On the other hand, there are Borel functions $f_{1}$ and $f_{2}$ such that $f_{1} \leqq f^{M} \leqq f_{2}$ on $X$ and $\int_{X}\left(f_{2}(x)-f_{1}(x)\right) m(d x)=0$. But, for any $h \in \boldsymbol{C}_{0}(X)$,

$$
\left(h, G_{n}\left(f_{2}-f_{1}\right)\right)=\left(\hat{G}_{n} h, f_{2}-f_{1}\right)=0
$$

yielding that $G_{n} f_{1}=G_{n} f_{2} m$-a.e. and hence q.e. owing to (vi). Therefore there is a Borel almost polar set $B_{n}$ such that $G_{n} f^{M}(x)=G_{n} f_{1}(x)$ for every $x \in X-B_{n}$. Put $B_{M}=B_{0} \cup \bigcup_{n=1}^{\infty} B_{n}$, then

$$
f^{M}(x)=\lim _{n \rightarrow \infty} n G_{n} f^{M}(x)=\lim _{n \rightarrow \infty} n G_{n} f_{1}(x), \quad x \in X-B_{M} .
$$

Consequently $f^{M}$ is Borel measurable on $X-B_{M}$. According to (vii), there is a Borel almost polar set $B \supset \bigcup_{M} B_{M}$ such that $X-B$ is $\boldsymbol{M}$-invariant. Then $f(x)=\lim _{M \rightarrow \infty} f^{M}(x)$ is Borel measurable on $X-B$, completing the proof.

(x) Let $\left\{f_{n}\right\}$ be a decreasing sequence of $\alpha$-excessive functions with limit $f$ and suppose that $f=0 \mathrm{~m}$-a.e. Then $f=0$ q.e.

This proposition corresponds to Blumenthal-Getoor [2; VI (3.2)]. The proof is quite the same. We do not know whether in our case every semipolar set is cosemipolar. But by making use of $(\mathbf{x})$ and following the same line as in Blumenthal-Getoor [2; VI (1.19)], we get

(xi) Each semipolar set is the sum of a cosemipolar set and an almost polar set.

(xii) For any Borel sets $A$ and $B$, we have

$$
\left(g, H_{\boldsymbol{A}}^{\alpha} H_{B}^{\alpha} G_{\alpha} h\right)=\left(\hat{H}_{B}^{\alpha} \hat{H}_{\boldsymbol{A}}^{\alpha} \hat{G}_{\alpha} g, h\right)
$$

for any non-negative Borel functions $g$ and $h$.

PROOF. This is a consequence of (2.2), Take non-negative $g$ and $h$ in $\boldsymbol{C}_{0}(X)$. Since $H_{B}^{\alpha} G_{\alpha} h$ (resp. $\hat{H}_{A}^{\alpha} \hat{G}_{\alpha} g$ ) is an $\alpha$-excessive (resp. $\alpha$-coexcessive) function, we have

$$
\begin{aligned}
\left(g, H_{A}^{\alpha} H_{B}^{\alpha} G_{\alpha} h\right) & =\lim _{\beta \rightarrow+\infty} \beta\left(g, H_{A}^{\alpha} G_{\beta} H_{B}^{\alpha} G_{\alpha} h\right) \\
& =\lim _{\beta \rightarrow+\infty} \beta\left(g, H_{A}^{\alpha} G_{\alpha}\left(I-(\beta-\alpha) G_{\beta}\right) H_{B}^{\alpha} G_{\alpha} h\right) \\
& =\lim _{\beta \rightarrow+\infty} \beta\left(\hat{H}_{B}^{\alpha} \hat{G}_{\alpha}\left(I-(\beta-\alpha) \hat{G}_{\beta}\right) \hat{H}_{A}^{\alpha} \hat{G}_{\alpha} g, h\right) \\
& =\lim _{\beta \rightarrow+\infty} \beta\left(\hat{H}_{B}^{\alpha} \hat{G}_{\beta} \hat{H}_{A}^{\alpha} \hat{G}_{\alpha} g, h\right)=\left(\hat{H}_{B}^{\alpha} \hat{H}_{A}^{\alpha} \hat{G}_{\alpha} g, h\right) .
\end{aligned}
$$

(xiii) Let $A$ be a Borel set. Denote by $A^{r}\left(r e s p .{ }^{r} A\right)$ the totality of regular (resp. coregular) points of $A$. Then ${ }^{r} A-A^{r}$ is written as 


$$
{ }^{r} A-A^{r}=N_{1}+N_{2}
$$

with a Borel semi-polar set $N_{1}$ and a nearly Borel almost polar set $N_{2}$. The same conclusion holds for $A^{r}-{ }^{r} A$.

ProOF. Since ${ }^{r} A$ is co-nearly Borel, there are Borel sets $\hat{A}^{\prime}$ and $\hat{A}^{\prime \prime}$ such that $\hat{A}^{\prime} \subset{ }^{r} A \subset \hat{A}^{\prime \prime}$ and $\hat{A}^{\prime \prime}-\hat{A}^{\prime}$ is almost copolar. There are also Borel sets $A^{\prime}$ and $A^{\prime \prime}$ such that $A^{\prime} \subset A^{r} \subset A^{\prime \prime}$ and $A^{\prime \prime}-A^{\prime}$ is almost polar. Put $F=\hat{A}^{\prime}-A^{\prime \prime}$, then $F$ is a Borel set, $F \subset^{r} A-A^{r}$ and the set $\left({ }^{r} A-A^{r}\right)-F$ is almost polar in view of (iv). By the preceding identity, we have

$$
\left(g, H_{\boldsymbol{A}}^{\alpha} H_{F}^{\alpha} G_{\alpha} h\right)=\left(\hat{H}_{F}^{\alpha} \hat{H}_{\boldsymbol{A}}^{\alpha} \hat{G}_{\alpha} g, h\right) .
$$

Since $F \cup^{r} F \subset^{r} A$, we see that $\hat{H}_{F}^{\alpha} \hat{H}_{A}^{\alpha} \hat{G}_{\alpha} g=\hat{H}_{F}^{\alpha} \hat{G}_{\alpha} g$. Hence, by (2.2), ( $g$, $\left.H_{A}^{\alpha} H_{F}^{\alpha} G_{\alpha} h\right)=\left(g, H_{F}^{\alpha} G_{\alpha} h\right)$. Now choose $h_{n}$ such that $G_{\alpha} h_{n} \uparrow 1$. We have $\left(g, H_{A}^{\alpha}{ }_{F}^{\alpha}\right)$ $=\left(g, e_{F}^{\alpha}\right)$ for every $g \in C_{0}(X)$. Using (vi) we get $e_{F}^{\alpha}=H_{A}^{\alpha} e_{F}^{\alpha}$ q. e. If $x \in F$, then $x \notin A^{r}$ and $H_{A}^{\alpha} e_{F}^{\alpha}(x) \leqq H_{A}^{\alpha} 1(x)<1$. Thus, there is an almost polar Borel set $N^{\prime}$ such that $e_{F}^{\alpha}(x)<1$ for $x \in N_{1}=F-N^{\prime} . \quad N_{1}$ is then a Borel semipolar set because $e_{N_{1}}^{\alpha}(x) \leqq e_{F}^{\alpha}(x)<1$ for $x \in N_{1}$. Now ${ }^{r} A-A^{r}=N_{1}+N_{2}$ with $N_{2}=$ $\left[\left({ }^{r} A-A^{r}\right)-F\right]+F \cap N^{\prime}$ is the desired expression.

(xiv) The following two conditions are equivalent.

$\left(\boldsymbol{C}_{1}\right)$ Each semipolar set is almost polar.

$\left(\boldsymbol{C}_{1}^{\prime}\right)$ A function is q.e. finely continuous if and only if it is q.e. cofinely continuous.

Proof. Assume the condition $\left(\boldsymbol{C}_{1}\right)$. Consider a q. e. finely continuous function $f$. By (ix), there is an almost polar Borel set $B$ such that $X_{0}=X-B$ is finely open and $f$ is Borel measurable and finely continuous on $X_{0}$. For a real number $a$, put $E_{a}=\left\{x \in X_{0} ; f(x)<a\right\}$. Since $X-E_{a}$ is finely closed,

$$
N_{a}={ }^{r}\left(X-E_{a}\right)-\left(X-E_{a}\right) \subset^{r}\left(X-E_{a}\right)-\left(X-E_{a}\right)^{r}
$$

which is almost polar on account of (xiii) and $\left(\boldsymbol{C}_{1}\right)$. Notice that $E_{a}-N_{a}=$ $E_{a}-{ }^{r}\left(X-E_{a}\right)$ is cofinely open. Choose an almost polar Borel set $N_{a}^{\prime} \supset N_{a}$ and set $\hat{B}_{0}=B \cup\left(\bigcup_{a \text { : rational }} N_{a}^{\prime}\right)$. By virtue of (vii), there exists an almost polar Borel set $\hat{B} \supset \hat{B}_{0}$ such that $X-\hat{B}$ is $\hat{M}$-invariant. Now, for any rational $a$, the set $\{x \in X-\hat{B} ; f(x)<a\}=E_{a}-\hat{B}$ is cofinely open because $E_{a}-\hat{B}=\left(E_{a}-N_{a}\right) \cap$ $(X-\hat{B})$ and both $E_{a}-N_{a}$ and $X-\hat{B}$ are cofinely open. This shows that $f$ is q. e. cofinely continuous.

Coming to the converse, fix a compact thin set $K$. We first note that

$$
P_{x}\left(\lim _{t \mid \sigma_{K}} e_{K}^{\alpha}\left(X_{t}\right)=1, \sigma_{K}<+\infty\right)=P_{x}\left(\sigma_{K}<+\infty\right) \quad \text { for } m \text {-a. e. } x \in X .
$$

To see this, let $\left\{G_{n}\right\}$ be open sets such that $G_{n} \supset \bar{G}_{n+1}$ and $\bigcap_{n} G_{n}=K$. Using (xii), we can easily see that $H_{G_{n}}^{\alpha} e_{K}^{\alpha}(x)=e_{K}^{\alpha}(x) m$-a. e. Since $K$ is thin, we then 
have $P_{x}\left(\sigma_{K}<+\infty\right)=P_{x}\left(\sigma_{G_{n}}<\sigma_{K}\right.$ for every $\left.n, \lim _{n} \sigma_{G_{n}}=\sigma_{K}, \sigma_{K}<+\infty\right)$ for $m$-a. e. $x \in X$. We arrive at (2.4) by virtue of [2; II (3.12)].

Now let us assume the condition $\left(\boldsymbol{C}_{1}^{\prime}\right)$ and prove that $K$ is almost polar. Put $B_{n}=\left\{x \in X ; e_{K}^{\alpha}(x) \geqq 1-\frac{1}{n}\right\}$, then (2.4) implies that $\sigma_{B_{n}}<\sigma_{K} P_{x}$-almost surely on $\left\{\sigma_{K}<+\infty\right\}$ for $m$-a. e. $x \in X$. Hence $H_{B_{n}}^{\alpha} H_{K}^{\alpha} f=H_{K} f m$-a. e. for any bounded Borel $f$. Using (xii) again, we finally get

$$
\left(\hat{H}_{K}^{\alpha} \hat{H}_{B_{n}}^{\alpha} g, h\right)=\left(\hat{H}_{K}^{\alpha} g, h\right)
$$

for bounded continuous $g$ and $m$-integrable bounded Borel $h$. On the other hand, the function $e_{K}^{\alpha}$ is, being $\alpha$-excessive, q. e. cofinely continuous by $\left(\boldsymbol{C}_{1}^{\prime}\right)$. On account of (ix), there is an $m$-negligible Borel set $N$ such that $X-N$ is $\hat{\boldsymbol{M}}$-invariant and each set $B_{n}-N$ is (relatively) cofinely closed in $X-N$. Fix a strictly positive bounded $m$-integrable function $h$. Rewriting (2.5) as $\left(\hat{H}_{K}^{\alpha} g, h\right)=\left(I_{X-N} \hat{H}_{K-N}^{\alpha} \hat{H}_{B_{n-N}}^{\alpha} g, h\right)$, we can observe that the measure $\pi(E)=$ $\left(\hat{H}_{K}^{\alpha} I_{E}, h\right)$ is concentrated on $B_{n}-N$. But $\cap B_{n}$ is empty and hence $\pi$ must be a zero measure. In particular, $\hat{e}_{K}^{\alpha}(x)=\hat{H}_{K}^{\alpha} 1(x)$ vanishes $m$-a. e., yielding that $K$ is almost polar.

\section{§3. An ergodic theorem.}

Let us assume the condition $\left(\boldsymbol{C}_{1}\right)$ throughout this section. We put

$$
K_{t} f(x)=\int_{0}^{t} T_{s} f(x) d s, \quad x \in X,
$$

for a bounded Borel function $f$ and $t>0$.

Our main theorem is this.

THEOREM 3.1. For any bounded Borel $f, g \in L^{1}(X ; m), g \geqq 0$, the ratio

$$
\frac{K_{t} f(x)}{K_{t} g(x)}
$$

converges, as $t \rightarrow+\infty$, to a finite limit q.e. on the set

$$
E_{g}=\bigcup_{t>0}\left\{x \in X ; K_{t} g(x)>0\right\} .
$$

This generalization of the Chacon-Ornstein ergodic theorem is an easy consequence of the following version of Brunel's lemma $[10 ; 2.7]$ and the assertion ( $\mathbf{v})$ of the preceding section.

LEMMA 3.1. Let $f$ be a bounded Borel function of $L^{1}(X ; m)$ and $A$ be $a$ Borel subset of

$$
\bigcap_{n=1}^{\infty}\left\{x \in X ; \sup _{t>n} K_{t} f(x)>0\right\}
$$


Then we have

$$
\left(\hat{e}_{A}, f\right) \geqq 0,
$$

where

$$
\hat{e}_{A}(x)=\hat{P}_{x}\left(\sigma_{A}<+\infty\right), \quad x \in X .
$$

We will have to use our condition $\left(\boldsymbol{C}_{1}\right)$ to prove Lemma 3.1. It is convenient to introduce here the notion of characteristics of the Markov process M. A non-negative finite valued function $c_{t}(x), t>0, x \in X$, is called a nonnegative characteristic if

$$
\begin{aligned}
& c_{t}(\cdot) \text { is universally measurable, } \\
& c_{s}(x)+T_{s} c_{t}(x)=c_{s+t}(x), \quad s, t>0, \quad x \in X, \\
& \lim _{t \downarrow 0} c_{t}(x)=0 .
\end{aligned}
$$

The difference of two non-negative characteristics is called merely a characteristic. $K_{t} f$ is a simple example of a characteristic. It is easy to see that any characteristic is right continuous in $t>0$. Furthermore any bounded characteristic is nearly Borel measurable and finely continuous in $x \in X$. The proof of this quite useful fact was given in E. B. Dynkin [7; Theorem 6.5].

PROOF OF THE IMPLICATION : LEMMA 3.1. $\Rightarrow$ THEOREM 3.1. We may assume, without loss of generality, that $f$ is also non-negative in the statement of Theorem 3.1. Since $K_{t} g$ is continuous in $t>0, E_{g}=\underset{\substack{r>0 \\ \text { rational }}}{\bigcup}\left\{x \in X ; K_{r} g(x)>0\right\}$, which is Borel measurable and finely open because of the corresponding properties of the function $K_{r} g$. Now let us consider the set

$$
N=\left\{x \in E_{g} ; \varlimsup_{t \rightarrow+\infty} \frac{K_{t} f(x)}{K_{t} g(x)}=+\infty\right\} .
$$

Then, for any $\lambda>0$,

$$
N \subset \bigcap_{n=1}^{\infty}\left\{\sup _{t \geqq n} K_{t}(f-\lambda g)(x)>0\right\} .
$$

By Lemma 3.1, we have

$$
+\infty>\left(\hat{e}_{N}, f\right) \geqq \lambda\left(\hat{e}_{N}, g\right) \geqq \frac{\lambda}{r}\left(\hat{K}_{r} \hat{e}_{N}, g\right)=\frac{\lambda}{r}\left(\hat{e}_{N}, K_{r} g\right) .
$$

$\lambda$ being arbitrary, we must have $\hat{e}_{N}=0 m$-a. e. on the set $\left\{x \in X ; K_{r} g(x)>0\right\}$ and hence $m$-a.e. on $E_{g}$. In view of $\S 2(\mathbf{v}), N$ is almost polar. In the same way, we see that the set

$$
N_{a b}=\left\{x \in E_{g} ; \varliminf_{t \rightarrow \infty} \frac{K_{t} f(x)}{K_{t} g(x)}<a, b<\varlimsup_{t \rightarrow \infty} \frac{K_{t} f(x)}{K_{t} g(x)}\right\}
$$

is also almost polar for any $a<b$.

q. e. d. 
From now on we will concentrate our attention on the proof of Lemma 3.1. We essentially follow the reasoning of A.M. Garsia [10; Chap. 2] and its refinement by P.A. Meyer [11; Theorem 6]. We have to prepare three lemmas (Lemma 3.2 3.4). The following lemma presents our version of Hopf's maximal ergodic inequality (in Garsia's form).

LEMMA 3.2. Let $c_{t}(x)$ be a bounded m-integrable characteristic and $h$ be $a$ positive number. We put $E_{h}=\left\{x \in X ; \sup _{n} c_{n h}(x)>0\right\}$. Then we have

$$
\left(I_{E_{h}} \cdot v, c_{h}\right) \geqq 0,
$$

where $v$ is an arbitrary bounded co-excessive function, namely, $v$ is non-negative bounded and $\hat{T}_{t} v \uparrow v$ as $t \downarrow 0$.

Proof. Consider the set

$$
E_{h}^{n}=\left\{x ; \max _{1 \leqq \nu \leqq n} c_{\nu h}(x)>0\right\}=\left\{x ; \max _{1 \leqq \nu \leqq n} c_{\nu h}^{+}(x)>0\right\} .
$$

For $x \in E_{h}^{n}$, we have

$$
c_{h}(x)+\max _{1 \leqq \nu \leqq n}\left(c_{(\nu+1) h}-c_{h}\right)^{+}(x) \geqq \max _{1 \leqq \nu \leqq n} c_{\nu h}^{+}(x) .
$$

Since $c_{t}$ is a characteristic,

$$
\left(c_{(\nu+1) h}-c_{h}\right)^{+}=\left(T_{h} c_{\nu h}\right)^{+} \leqq T_{h} c_{\nu h}^{+}
$$

and hence

$$
\max _{1 \leqq \nu \leqq n}\left(c_{(\nu+1) h}-c_{h}\right)^{+} \leqq T_{h} \max _{1 \leqq \nu \leqq n} c_{\nu h}^{+}
$$

Therefore we have

$$
\begin{aligned}
\left(I_{E_{h}^{n}} \cdot v, c_{h}\right) & \geqq\left(I_{E_{h}^{n}} \cdot v, \max _{1 \leqq \nu \leqq n} c_{\nu h}^{+}-T_{h}\left(\max _{1 \leqq \nu \leqq n} c_{\nu h}^{+}\right)\right) \\
& \geqq\left(v, \max _{1 \leqq \nu \leqq n} c_{\nu h}^{+}-T_{h}\left(\max _{1 \leqq \nu \leqq n} c_{\nu h}^{+}\right)\right) \\
& =\left(v-\widehat{T}_{h} v, \max _{1 \leqq \nu \leqq n} c_{\nu h}^{+}\right) \geqq 0 .
\end{aligned}
$$

Letting $n$ tend to infinity, we get (3.10),

Consider next a Borel set $A \subset X$ and a constant $T>0$. We put

$$
\begin{aligned}
& { }^{(0)} T_{t} g(x)=E_{x}\left(g\left(X_{t}\right) ; t<\sigma_{A}\right) \\
& { }^{(0)} K_{t} g(x)=\int_{0}^{t}{ }^{(0)} T_{s} g(x) d s \\
& H_{\sigma_{A} \wedge T} g(x)=E_{x}\left(g\left(X_{\sigma_{A} \wedge T}\right)\right) .
\end{aligned}
$$

LEMMA 3.3. Let $f$ be a bounded m-integrable Borel function and $d_{t}^{f}$ be

$$
d_{t}^{f}(x)=K_{t} f(x)-{ }^{(0)} K_{T} f(x)+T_{t} \cdot{ }^{(0)} K_{T} f(x), \quad t>0, \quad x \in X .
$$

Then 
(i) $d_{t}^{f}$ is a bounded m-integrable characteristic.

(ii) $d_{t}^{f}$ satisfies the equality

$$
\left(g, d_{t}^{f}\right)=\left(\hat{H}_{\sigma_{A} \wedge T} \hat{K}_{t} g, f\right)
$$

for any bounded Borel function $g$.

PROOF. We write $d_{t}^{f}$ simply as $d_{t}$.

(i) $d_{t}$ is bounded and universally measurable. It follows from (1.1) that $\left\|T_{t} f\right\|_{L^{1}} \leqq\|f\|_{L^{1}}$ for any Borel $f \in L^{1}(X ; m)$. Hence $d_{t} \in L^{1}(X ; m)$. The equality (3.8) for $d_{t}$ is easily verified. Let us verify (3.9). Denote $\sigma_{A} \wedge T$ by $\sigma$. Since ${ }^{(0)} K_{T} f(x)=E_{x}\left(\int_{0}^{\sigma} f\left(X_{s}\right) d s\right)$, we have $T_{t}{ }^{(0)} K_{T} f(x)=E_{x}\left(\int_{t}^{t+\sigma\left(\theta_{t}(\omega)\right.} f\left(X_{s}\right) d s\right)$, $\theta_{t}$ being the shift of the sample path $\omega:\left(\theta_{t} \omega\right)_{s}=\omega_{t+s}, s>0$. Hence we have $d_{t}(x)=E_{x}\left(\int_{\sigma}^{t+\sigma\left(\theta_{t}(\omega)\right.} f\left(X_{s}\right) d s\right)$, which tends to zero as $t \downarrow 0$ because $t+\sigma\left(\theta_{t}\right) \rightarrow \sigma(t \downarrow 0)$.

(ii) We introduce $\alpha$-order quantities :

$$
\begin{array}{ll}
\hat{T}_{t}^{\alpha} g(x)=e^{-\alpha t} \hat{T}_{t} g(x), & { }^{(0)} \hat{T}_{t}^{\alpha} g(x)=e^{-\alpha t(0)} \hat{T}_{t} g(x), \\
{ }^{(0)} \hat{K}_{T}^{\alpha} g(x)=\int_{0}^{T}{ }^{(0)} \hat{T}_{s}^{\alpha} g(x) d s, & \hat{H}_{\sigma}^{\alpha} g(x)=\hat{E}_{x}\left(e^{-\alpha \sigma} g\left(X_{\sigma}\right)\right) .
\end{array}
$$

Since ${ }^{(0)} \hat{K}_{T}^{\alpha} g(x)=\hat{E}_{x}\left(\int_{0}^{\sigma} e^{-\alpha s} g\left(X_{s}\right) d s\right)$, we have by Dynkin's formula

$$
\hat{H}_{\sigma}^{\alpha} \hat{G}_{\alpha} g=\hat{G}_{\alpha} g-{ }^{(0)} \hat{K}_{T}^{\alpha} g \text {. }
$$

Replacing the function $g$ in (3.13) by $\hat{T}_{t}^{\alpha} g$ and subtracting the resulting equality from (3.13), we have $\hat{H}_{\sigma}^{\alpha} \hat{K}_{t}^{\alpha} g=\hat{K}_{t}^{\alpha} g-{ }^{(0)} \hat{K}_{T}^{\alpha} g+{ }^{(0)} \widehat{K}_{T}^{\alpha} \hat{T}_{t}^{\alpha} g$. Letting $\alpha$ tend to zero,

$$
\hat{H}_{\sigma} \hat{K}_{t} g=\hat{K}_{t} g-{ }^{(0)} \hat{K}_{T} g+{ }^{(0)} \hat{K}_{T} \hat{T}_{t} g .
$$

Taking the duality relations (1.1) and (2.1) of $T_{t}$ and ${ }^{(0)} T_{t}$ into consideration, we finally get

$$
\left(\hat{H}_{\sigma} \hat{K}_{t} g, f\right)=\left(g, K_{t} f-{ }^{(0)} K_{T} f+T_{t}{ }^{(0)} K_{T} f\right)=\left(g, d_{t}\right) .
$$

Let us write as $A_{1} \subset A_{\mathbf{q} . e}$ if $A_{1}-A_{2}$ is almost polar.

LEMMA 3.4. Let $f$ be a bounded m-integrable Borel function and $A$ be $a$ Borel subset of

$$
\bigcap_{n}\left\{x \in X ; \sup _{t>n} K_{t} f(x)>0\right\} .
$$

Fix a constant $T>0$ and consider the characteristic $d_{t}^{f}$ of Lemma 3.3 defined for the present $f, A$ and $T$.

Then, for any $\varepsilon>0$,

$$
A \subset\left\{x \in X ; \sup _{t>0}\left(\varepsilon K_{t}|f|(x)+d_{t}^{f}(x)\right)>0\right\} .
$$


Proof. From (3.9),

Observe that

$$
d_{t}^{f}(x)+T_{t} K_{T}|f|(x) \geqq K_{t} f(x)-{ }^{(0)} K_{T} f(x) .
$$

$$
\lim _{t \rightarrow \infty} \frac{T_{t} K_{T}|f|(x)}{K_{t}|f|(x)}=0, \quad x \in X,
$$

defining $0 / 0$ to be 0 by convention. Hence, for any $\varepsilon>0$,

$$
d_{t}^{f}(x)+\varepsilon K_{t}|f|(x) \geqq K_{t} f(x)-{ }^{(0)} K_{T} f(x)
$$

for every $t$ greater than some $t_{0}=t_{0}(x)>0$. On the other hand, the condition $\left(\boldsymbol{C}_{1}\right)$ implies

$$
P_{x}\left(\sigma_{A}=0\right)=1 \quad \text { for } \text { q. e. } x \in A \text {. }
$$

This is because $A-A^{r}$ is semipolar (c. f. [2; II (3.3)] $)$ and hence almost polar by $\left(\boldsymbol{C}_{1}\right)$. Therefore we have

$$
{ }^{(0)} K_{T} f(x)=E_{x}\left(\int_{0}^{\sigma_{A} \wedge T} f\left(X_{s}\right) d s\right)=0 \quad \text { for } \text { q. e. } x \in A,
$$

arriving at the desired inclusion (3.15),

We are now ready to prove Lemma 3.1.

PROOF of LEMMA 3.1. Let $f$ be a bounded Borel function of $L^{1}(X ; m)$. Consider any compact subset $A$ of (3.14), Then, by Lemma 3.4, we have the inclusion (3.15) with an arbitrarily fixed $T>0$ and $\varepsilon>0$.

The characteristic $d_{t}^{f}$ satisfies the relation (3.12) which can be rewritten as follows:

$$
\left(g, d_{t}^{f}\right)=\left(\hat{H}_{A}^{T} \hat{K}_{t} g, f\right)+\left(\hat{Q}_{T} \hat{K}_{t} g, f\right)
$$

where

$$
\left\{\begin{array}{l}
\hat{H}_{A}^{T} h(x)=\hat{E}_{x}\left(h\left(X_{\sigma_{A}}\right) ; \sigma_{A}<T\right) \\
\hat{Q}_{T} h(x)=\hat{E}_{x}\left(h\left(X_{T}\right) ; T<\sigma_{A}\right) .
\end{array}\right.
$$

Let us put

$$
c_{t}(x)=\varepsilon K_{t}|f|(x)+d_{t}^{f}(x),
$$

which is a bounded $m$-integrable characteristic. We then define

$$
E_{\varepsilon, l}=\left\{x \in X ; \max _{\nu} c_{\nu / 2}(x)>0\right\}
$$

for positive integer $l$. By virtue of Lemma 3.2, we have $\left(I_{E_{\varepsilon, l}} \cdot v, c_{1 / 2} l\right) \geqq 0$ for any bounded co-excessive function $v$. Combining this with (3.17) and (3.19), we get

$$
\varepsilon\left(\hat{K}_{1 / 2} l v,|f|\right)+\left(\hat{Q}_{T} \hat{K}_{1 / 2} l v,|f|\right)+\left(\hat{H}_{A}^{T} \hat{K}_{1 / 2} l\left(I_{E_{\varepsilon, l}} \cdot v\right), f\right) \geqq 0 .
$$

Multiplying $2^{l}$ and letting $l$ tend to infinity, 


$$
\varepsilon(v,|f|)+\left(\hat{Q}_{T} v,|f|\right)+\varlimsup_{l \rightarrow \infty} 2^{l}\left(\hat{H}_{A}^{T} \widehat{K}_{1 / 2} l\left(I_{E_{\varepsilon, l}} \cdot v\right), f\right) \geqq 0 .
$$

Now look at the last term of the left hand side of this inequality. Recalling Lemma 3.4, we see that, there is an almost polar set $N_{1}$ such that $A-N_{1} \sqsubseteq \bigcup_{l=1}^{\infty} E_{\varepsilon, l}$. Furthermore we can find almost polar sets $N_{2}^{l}$ such that each set $E_{\varepsilon, l}-N_{2}^{l}$ is cofinely open, because $c_{t}(x)$ is finely continuous and hence q.e. cofinely continuous by virtue of the condition $\left(\boldsymbol{C}_{1}^{\prime}\right)$ which is equivalent to $\left(\boldsymbol{C}_{1}\right)$ $\left(\S 2\right.$, (xiv)). Select then an almost polar set $N_{2} \supset \bigcup_{l=1}^{\infty} N_{2}^{l}$ such that $X-N_{2}$ is $M$-invariant according to $\S 2$ (vii). It is easy to see that each $E_{\varepsilon, l}-N_{2}$ is cofinely open. Thus

$$
\lim _{l \rightarrow \infty} 2^{l} \hat{K}_{1 / 2} l\left(I_{E_{\varepsilon, l}} \cdot v\right)(x)=v(x), \quad x \in A-N_{1} \cup N_{2} .
$$

Since the signed measure $f \cdot \hat{H}_{A}^{T}(E)=\left(\hat{H}_{A}^{T} I_{E}, f\right)$ is concentrated on the compacturn $A$ and does not charge on any almost polar set, we obtain

$$
\varepsilon(v,|f|)+\left(\hat{Q}_{T} v,|f|\right)+\left(\hat{H}_{\boldsymbol{A}}^{T} v, f\right) \geqq 0 .
$$

Letting $\varepsilon$ tend to zero, $\left(\hat{Q}_{T} v,|f|\right)+\left(\hat{H}_{A}^{T} v, f\right) \geqq 0$. Finally put $v=\hat{e}_{A}$ and let $T$ tend to infinity. Observe that $\hat{Q}_{T} \hat{e}_{A}(x)=\hat{P}_{x}\left(\sigma_{A}\left(\theta_{T} \omega\right)<+\infty, T<\sigma_{A}\right)=\hat{P}_{x}(T<$ $\left.\sigma_{A}<+\infty\right) \rightarrow 0, T \rightarrow \infty$. Using again the property of the measure $f \cdot \hat{H}_{\boldsymbol{A}}^{T}$ stated just before (3.22) and recalling (3.16), we are led to $\lim _{T \rightarrow \infty}\left(\hat{H}_{\boldsymbol{A}}^{T} \hat{e}_{\boldsymbol{A}}, f\right)=\lim _{T \rightarrow \infty}\left(\hat{H}_{\boldsymbol{A}}^{T} 1, f\right)$ $=\left(\hat{e}_{A}, f\right)$, arriving at the desired inequality (3.5).

\section{§4. An ergodic decomposition of the state space.}

Let $\Phi$ be an $m$-integrable bounded continuous function strictly positive on $X$ and let us put

$$
\left\{\begin{array}{l}
C=\{x \in X ; G \Phi(x)=+\infty\} \\
D=\{x \in X ; G \Phi(x)<+\infty\}
\end{array}\right.
$$

where $G \Phi(x)=\lim _{t \rightarrow \infty} K_{t} \Phi(x)$. We call $C$ (resp. $D$ ) the conservative (resp. dissipative) part of $X$. If we assume the condition $\left(\boldsymbol{C}_{1}\right)$, then Theorem 3.1 holds and hence the decomposition $X=C+D$ does not depend on the choice of such a $\Phi$ up to an almost polar set.

In order to study some more about this decomposition, let us assume the following conditions throughout this section:

$\left(C_{2}\right)$ A set is semipolar if and only if it is almost polar,

$\left(C_{3}\right)$ Each point $x \in X$ is either polar or stable,

where we say a point $x \in X$ to be stable if almost all sample paths starting at $x$ stay there during some initial time intervals. 
The condition $\left(\boldsymbol{C}_{2}\right)$ is the same as $\left(\boldsymbol{C}_{1}\right)$ coupled with the assumption that the measure $m$ is a reference measure for the process $M(\S 2$, (viii)). In particular, $\left(\boldsymbol{C}_{2}\right)$ is met when $\boldsymbol{M}=\hat{\boldsymbol{M}}$ and the resolvent is absolutely continuous with respect to $m$. Under $\left(\boldsymbol{C}_{2}\right)$, polarity, semipolarity and almost polarity become the equivalent notions. $\left(C_{3}\right)$ corresponds to the condition $\left(L^{\prime}\right)$ of J.L. Doob [6], In [6] the notion of a (fine) quasinull set was principally used, which is however equivalent, under $\left(\boldsymbol{C}_{2}\right)$ and $\left(\boldsymbol{C}_{3}\right)$, to the polarity of the set (see $[6 ; \S 8]$ ).

A theorem of Doob [6; Theorem 8.1] states that, if $\boldsymbol{M}$ has a reference measure, the fine topology relative to $M$ has the quasi Lindelöf property, that is, every union of finely open sets is equal, up to a quasinull set, to a countable subunion. Therefore, under the present assumptions $\left(\boldsymbol{C}_{2}\right)$ and $\left(\boldsymbol{C}_{3}\right)$, we have

LEMMA 4.1. Every union of finely open sets is a sum of a countable subunion and a polar set.

Now following Azema, Kaplan-Duflo and Revuz [1], we call a point $x \in X$ finely transient if there is a nearly Borel fine neighbourhood $V$ of $x$ such that

$$
P_{x}\left(\varlimsup_{t \rightarrow \infty} I_{V}\left(X_{t}\right)=1\right)=0 \text {. }
$$

Otherwise $x$ is called finely recurrent. A point $x \in X$ is finely transient if and only if there is a non-negative bounded Borel function $f$ such that

$$
0<G f(x)<+\infty
$$

is valid ([1; Proposition 5$])$. We will soon use this criterion. Denote by $T$ (resp. $R$ ) the set of all transient (resp. recurrent) points of $X$.

THEOREM 4.1. $D$ is a subset of $T$ and the difference $N=T-D$ is polar. Furthermore $R(=C-N)$ is $\boldsymbol{M}$-invariant.

Proof. Since $G \Phi$ is strictly positive everywhere together with $\Phi$, we clearly have $D \subset T$.

Observe that, from the relation (3.8) of the characteristic, we have $K_{t} f+T_{t} G f=G f$ for any non-negative bounded Borel $f$, which in turn tells us that $G f$ is excessive and hence finely continuous. For each point $x \in T$, let us associate a non-negative bounded Borel function $f_{x}$ such that (4.3) holds. We may further assume that $f_{x}$ is $m$-integrable. By the above observation, the set $U(x)=\left\{y ; 0<G f_{x}(y)<+\infty\right\}(\subset T)$ is a fine neighbourhood of $x \in T$. By Lemma 4.1, there exist points $x_{i} \in T, i=1,2, \cdots$, and a polar set $N$ such that

$$
T=\left(\bigcup_{i=1}^{\infty} U\left(x_{i}\right)\right) \cup N
$$

Applying Theorem 3.1 to $\Phi$ and $f_{x_{i}}$, we see that each set $C \cap U\left(x_{i}\right)$ is polar. 
Therefore $N=C \cap T$ is polar in view of (4.4). Since $N$ is polar, it remains to show

$$
P_{x}\left(\sigma_{D}<+\infty\right)=0, \quad x \in R .
$$

Assume that (4.5) is false, then there are a point $x \in R$ and a set

$$
\widetilde{D}=\{x \in X ; \delta<\Phi(x), G \Phi(x)<M\} \quad(\subset D)
$$

with some $0<\delta<M<+\infty$ such that

$$
P_{x}\left(\sigma_{\tilde{D}}<+\infty\right)>0 \text {. }
$$

Now put $\Psi(y)=\Phi(y) \cdot I_{\tilde{D}}(y), y \in X$, then

$$
G_{\alpha} \Psi(x)=E_{x}\left(e^{-\alpha \sigma \tilde{D}} G_{\alpha} \Psi\left(X_{\sigma_{\tilde{D}}}\right)\right) .
$$

Since $G \Phi$ is right continuous along sample paths, (4.8) implies $G_{\alpha} \Psi(x)$ $\leqq E_{x}\left(G \Phi\left(X_{\sigma_{\tilde{D}}}\right)\right) \leqq M$ yielding $G \Psi(x) \leqq M$. Furthermore (4.7) and (4.8) imply

$$
G \Psi(x) \geqq \delta E_{x}\left(e^{-\alpha \sigma \tilde{D}} G_{\alpha} I_{\tilde{D}}\left(X_{\sigma_{\tilde{D}}}\right)\right)>0,
$$

because, $\tilde{D}$ being finely open, $G_{\alpha} I_{\tilde{D}}(y)$ is strictly positive for every $y \in \tilde{D} \cup \tilde{D}^{r}$ (the support of $X_{\sigma_{\tilde{D}}}$ ). Thus we get $0<G \Psi(x) \leqq M$ contradiction to the fact that $x$ is finely recurrent.

\section{References}

[1] J. Azema, M. Kaplan-Duflo and D. Revuz, Classes récurrentes d'un processus de Markov, Seminaire de Probabilités II, Lecture notes in Mathematics, 51, Springer, Berlin, 1968.

[2] R.M. Blumenthal and R.K. Getoor, Markov processes and potential theory, Academic press, New York, 1968.

[3] A. Brunel, Sur un lemme ergodique voisin du lemme de E. Hopf, et sur une de ses applications, Compt. Rend., 256 (1963), 5481-5484.

[4] L. Carleson, Selected problems on exceptional sets, Van Nostrand, New York, 1967.

[5] R. V. Chacon and D. Ornstein, A general ergodic theorem, Illinois J. Math., 4 (1960), 153-160.

[6] J.L. Doob, Applications to analysis of a topological definition of smallness of a set, Bull. Amer. Math. Soc., 72 (1966), 579-600.

[7] E.B. Dynkin, Markov processes, Springer, Berlin, 1965.

[8] M. Fukushima, Dirichlet spaces and strong Markov processes, Trans. Amer. Math. Soc., 162 (1971), 185-224.

[9] M. Fukushima, On absolute continuity of symmetric transition probabilities, J. Math. Kyoto Univ., 12 (1972), 431-450.

[10] A. Garsia, Topics in almost everywhere convergence, Markham, Chicago 1970.

[11] P.A. Meyer, Travaux de H. Rost en théorie du balayage, Seminaire de Probabilités V, Lecture notes in Mathematics, 191, Springnr, Berlin, 1970. 
[12] S.C. Port and C. J. Stone, Infinitely divisible processes and their potential theory, Ann. Inst. Fourier, 21 (1971), 157-275.

[13] M. G. Sur, Ergodic theorems for a class of Markov processes, Teor. Veroyatnost. i Primenen., 12 (1967), 443-454.

[14] T. Watanabe, Some potential theory of processes with stationary independent increments by means of the Schwartz distribution theory, J. Math. Soc. Japan, 24 (1972), 213-231.

Masatoshi Fukushima

Department of Mathematics Faculty of Science

Osaka University

Toyonaka, Osaka

Japan 